\title{
THE CONJUGACY PROBLEM FOR HNN EXTENSIONS WITH INFINITE CYCLIC ASSOCIATED GROUPS
}

\author{
K. J. HORADAM AND G. E. FARR \\ (Communicated by Ronald M. Solomon)
}

\begin{abstract}
Under suitable recursive conditions, the conjugacy problem for HNN extensions of the form $\left\langle G, t_{i}, i \in I: x^{k_{i}}=t_{i}^{-1} x^{l_{i}} t_{i}, k_{i}, l_{i} \geq 1, i \in I\right\rangle$ is solvable if and only if the inverse subsemigroup generated by $\left\{\left(k_{i}, l_{i}\right), i \in\right.$ I\} has solvable extended word problem in the semigroup

$$
S=(\mathbf{N} \times \mathbf{N},(a, b)(c, d)=(a c / \operatorname{gcd}(b, c), b d / \operatorname{gcd}(b, c))) .
$$

Furthermore, $S$ is isomorphic to the direct sum of countably many copies of the bicyclic semigroup, which has a central place in the theory of inverse semigroups.

This new approach to the conjugacy problem is used to determine several classes of HNN extensions with infinitely many stable letters and solvable conjugacy problem.
\end{abstract}

\section{INTRODUCTION} tions

The conjugacy problem (CP) for the class of HNN extensions with presenta-

$$
G^{*}=\left\langle G, t_{i}, i \in I: x^{k_{i}}=t_{i}^{-1} x^{l_{i}} t_{i}, k_{i}, l_{i} \in \mathbb{Z}, \quad i \in I\right\rangle
$$

where $G$ has solvable CP, $I$ is recursive, $x$ is a known element of infinite order in $G$, and $\langle x\rangle$ has solvable extended word problem in $G$, has been considered by several authors. In the case that card $I=1$, an example with unsolvable $C P$ is due to Lockhart [8]. In the case that $I$ is finite and the further conditions " $G$ is recursively presented" and " $x$ is central in $G$ " are imposed, the CP for $G^{*}$ was proved in some cases to be solvable by Horadam [5]; recently Lockhart [9] has proved it solvable in all cases by reformulating the CP in terms of the word problem in a commutative semigroup. An earlier reformulation of the $\mathrm{CP}$ in terms of the word problem in a commutative semigroup arose for other families of extensions (1) with finite $I$ and solvable CP, studied by Anshel [1, 2]

Received by the editors July 8, 1992; presented to the 36th Annual Conference of the Australian Mathematical Society on July 6, 1992.

1991 Mathematics Subject Classification. Primary 20F10, 20E06, 20 M18.

Key words and phrases. Conjugacy problem, HNN extension, extended word problem, inverse semigroup, bicyclic semigroup. 
and Anshel and McAloon [3], in which the exponents $k_{i}, l_{i}, i \in I$, are required to satisfy a relative primality condition.

We will concentrate on HNN extensions of the following kind.

Assumptions 1.1. $G^{*}$ satisfies the conditions of (1) and, additionally,

$G$ is recursively presented,

$x$ is central in $G$,

the exponents are positive: $G^{*}=\left\langle G, t_{i}, i \in I: x^{k_{i}}=t_{i}^{-1} x^{l_{i}} t_{i}, k_{i}, l_{i} \in\right.$ $\mathbb{N}, i \in I\rangle$,

the exponent maps $k, l: I \rightarrow \mathbb{N}$ are partially recursive, uniformly given from $I$,

and the exponent set $\left\{k_{i}, l_{i}, i \in I\right\}$ is recursive.

We prove in $\S 2$ that for $G^{*}$ satisfying Assumptions 1.1 the CP is equivalent to the extended word problem (EWP) for corresponding subsemigroups of a particular highly structured noncommutative inverse semigroup $S$. This formulation seems to be relatively tractable, and we obtain positive solutions to the $\mathrm{CP}$ for cases where the exponent pairs generate a subsemigroup of idempotents or else satisfy a relative primality condition. However, Assumptions 1.1 are insufficient to ensure that $G^{*}$ has solvable CP.

In $\S 3$ we identify $S$ in terms of a known inverse semigroup $B$, the bicyclic semigroup, which has ground set $(\mathbb{N} \cup\{0\}) \times(\mathbb{N} \cup\{0\})$ and binary operation $(a, b)(c, d)=(a+c-\min (b, c), b+d-\min (b, c))$. We prove that $S$ is isomorphic to $\bigoplus_{\omega} B$, the direct sum of countably infinitely many bicyclic semigroups.

Knowledge of the structure of the inverse subsemigroups of $B$ permits us to give a further positive solution to the $\mathrm{CP}$ for infinite $\mathrm{HNN}$ extensions where the exponents are powers of a common (known) base (in contrast with the relative primality case mentioned above).

\section{Edgepath Semigroups and the Conjugacy Problem}

In this section we translate the CP for the HNN extensions satisfying (1.1) into the EWP for "edgepath" semigroups.

Denote the greatest common divisor of any pair $a, b \in \mathbb{N}$ by $a \wedge b$.

Definition 2.1. Define a semigroup $S=(\mathbb{N} \times \mathbb{N}, \odot)$ by

$$
(a, b) \odot(c, d)=(a c /(b \wedge c), b d /(b \wedge c)), \quad \forall(a, b),(c, d) \in \mathbb{N} \times \mathbb{N} .
$$

The semigroup $S$ has $\{(a, a): a \in \mathbb{N}\}$ as its set of idempotents and has an identity $(1,1)$. Clearly $S$ is inverse, with $(a, b)^{-1}=(b, a)$.

By iteration, a formula for a product of $n$ elements in $S$ may be developed: if $\left(x_{i}, y_{i}\right) \in S, 1 \leq i \leq n$, then

$$
\left(x_{1}, y_{1}\right) \odot\left(x_{2}, y_{2}\right) \odot \cdots \odot\left(x_{n}, y_{n}\right)=\left(x_{1} g_{1} / g, y_{n} g_{n} / g\right),
$$

where

$$
g_{i}=\prod_{j=2}^{i} y_{j-1} \prod_{j=i+1}^{n} x_{j}, \quad 1 \leq i \leq n, \quad g=g_{1} \wedge g_{2} \wedge \cdots \wedge g_{n}
$$


Definition 2.2. Let $J, 1 \leq$ card $J \leq \omega$, be an index set, and let $\phi: J \rightarrow S$ be a set mapping. The inverse subsemigroup of $S$ generated by the set $\phi(J)$ together with its inverses will be termed an edgepath semigroup and denoted $P(J, \phi)$.

The edgepath semigroups are so named by us because they are specializations of the "edgepath groupoids" we define from a graph in the following fashion. If $D=(V, E)$ is a directed connected graph with vertex set $V$ and edge set $E$, then a set mapping $\phi: E \rightarrow S$ extends to a mapping from the set of nontrivial edgepaths in the (Brandt) groupoid $\mathscr{P}(D)$ of edgepaths of $D$ by the rules: $\phi(\bar{e})=(\phi(e))^{-1}$ and $\phi\left(e_{1} e_{2} \cdots e_{n}\right)=\phi\left(e_{1}\right) \odot \phi\left(e_{2}\right) \odot \cdots \odot \phi\left(e_{n}\right)$. The image $\mathscr{P}(D, \phi)$ of this set under $\phi$ is then a set with an associative partial multiplication, which, by a slight abuse of terminology, we call an edgepath groupoid. When $V$ is a single vertex $\{\cdot\}$, so that the edges indexed by $E$ are directed loops, then all products in an edgepath groupoid $\mathscr{P}((\{\cdot\}, E), \phi)$ are defined, so it is the edgepath semigroup $P(E, \phi)$.

To each fundamental group $G^{*}(\mathscr{G}, D)$ of a graph of groups $(\mathscr{G}, D)$ (see, e.g., [5] or [11] for any unfamiliar definitions), of the particular form

$$
\begin{aligned}
& G^{*}(\mathscr{G}, D)=\left\langle G_{v}, v \in V, t_{e}, e \in E:\right. \\
&\left.x_{t e}^{k_{e}}=t_{e}^{-1} x_{s e}^{l_{e}} t_{e}, e \in E, t_{e}=1, e \in T\right\rangle,
\end{aligned}
$$

where $T$ is a spanning tree of $D, x_{v}$ is an element of infinite order in $G_{v}$ and $\left\{k_{e}, l_{e}, e \in E\right\} \subseteq \mathbb{N}$, we can associate an edgepath groupoid $\mathscr{P}\left(D, \phi_{G^{*}(\mathscr{G}, D)}\right)$ by defining the mapping $\phi_{G^{*}(\mathscr{G}, D)}: E \rightarrow S$ to be $\phi_{G^{*}(\mathscr{G}, D)}(e)=\left(k_{e}, l_{e}\right)$.

In particular, when $D=(\{\cdot\}, I)$, the fundamental group $G^{*}(\mathscr{G}, D)$ of $(4)$ is an HNN extension $G^{*}$, and the associated edgepath semigroup is $P\left(I, \phi_{G^{*}}\right)=$ $\mathscr{P}\left(D, \phi_{G^{*}}\right)$.

In $[5,3.3]$, the $\mathrm{CP}$ for a $G^{*}(\mathscr{G}, D)$ of the form (4) satisfying the recursive conditions $[5,2.1,2.3]$ is shown to be solvable if and only if a derived recursively enumerable (r.e.) set is recursive. This set is in fact $\mathscr{P}\left(D, \phi_{G^{*}(\mathscr{G}, D)}\right)$. We only prove this for the HNN extensions.

Theorem 2.3. If $G^{*}$ satisfies Assumptions 1.1, $G^{*}$ has solvable CP if and only if $P\left(I, \phi_{G^{*}}\right)$ has solvable EWP in $S$.

Proof. If $G^{*}$ satisfies Assumptions 1.1 then it satisfies the recursive conditions $[5,2.1,2.3]$, and [5, 3.3] applies. Let $A=\left\{\left(k_{i}, l_{i}\right),\left(l_{i}, k_{i}\right), i \in I\right\}$. Comparison of the proof of $[5,3.3]$ with formulas (2) and (3) proves that $G^{*}$ has solvable $\mathrm{CP}$ if and only if the r.e. set

$$
\begin{array}{r}
\left\{(x, y)=\left(x_{1}, y_{1}\right) \odot\left(x_{2}, y_{2}\right) \odot \cdots \odot\left(x_{n}, y_{n}\right), n \geq 1:\right. \\
\left.\left(x_{j}, y_{j}\right) \in A, 1 \leq j \leq n\right\},
\end{array}
$$

is recursive. By definition, this r.e. set is the edgepath semigroup $P\left(I, \phi_{G^{*}}\right)$. Again by definition, it has solvable EWP in $S$ (as a subsemigroup) if and only if it is a recursive subset of $S$.

Assumptions 1.1 imply $\phi_{G^{*}}(I)$ is an r.e. generating set for $P\left(I, \phi_{G^{*}}\right)$. However, this need not be so for an arbitrary edgepath semigroup, and in [4] we focus on the more general question: 
When is the EWP for an edgepath semigroup $P(I, \phi)$ solvable?

We know the answer when $I$ is finite. Without appealing to the recursive set formulation of [5, 3.3], Lockhart [9] shows that, if $D$ is finite, the CP for those $G^{*}(\mathscr{G}, D)$ satisfying the recursive conditions $[5,2.1,2.3]$ is solvable.

Proposition 2.4 [9]. Let $D$ be finite, and let $G^{*}(\mathscr{G}, D)$ be as in (4), where $(\mathscr{G}, D)$ is a graph of recursively presented groups, $G_{v}$ has solvable $C P, x_{v}$ is central in $G_{v}$, and $\left\langle x_{v}\right\rangle$ has solvable extended word problem in $G_{v}$ for all $v \in V$. Then the CP for $G^{*}(\mathscr{G}, D)$ is solvable.

Corollary 2.5. If I is finite and $\phi: I \rightarrow S$ is a set mapping, the EWP for the edgepath semigroup $P(I, \phi)$ is solvable.

Proof. Define an HNN extension $G^{*}=G^{*}(\mathscr{G},(\{\cdot\}, I))$ as in (4), by equating the vertex group and all edge groups to the same infinite cyclic group $\langle x:\rangle$ and equating the exponent pair $\left(k_{i}, l_{i}\right)$ to $\phi(i)$ for all $i \in I$. Then $G^{*}$ has solvable CP by Proposition 2.4 and $\phi_{G^{*}}=\phi$; hence, $P(I, \phi)$ is a recursive set by Theorem 2.3 .

By Proposition 2.4 and the recursiveness of $I$,

we assume from now on in Assumptions 1.1 that $I=\mathbb{N}$,

without loss of generality. The following example shows that not every $G^{*}$ satisfying the assumptions has solvable CP.

Lemma 2.6. There exists an HNN extension $G^{*}$ satisfying Assumptions 1.1 which has unsolvable CP.

Proof. Let $G=\langle x:\rangle$, let $\left(p_{i}\right)_{i=1}^{\infty}$ be the sequence of primes $2,3, \ldots$, and let $\psi: \mathbb{N} \rightarrow \mathbb{N}$ be a one-to-one recursive function such that $\operatorname{Im} \psi$ is r.e. but not recursive. Define $k, l$ by $k(2 i-1)=l(2 i-1)=p_{i}, k(2 i)=1, l(2 i)=p_{\psi(i)}$, $i \in \mathbb{N}$. Every idempotent in $S$ arises as a product of odd-index generators, but any nonidempotent in $P\left(\mathbb{N}, \phi_{G^{*}}\right)$ must have a component with at least one even-index factor $l(2 i)$. It is not possible to decide if a nonidempotent element $(x, y)$ of $S$ is of this form, since the prime decomposition of either $x$ or $y$ must be $\prod_{j=1}^{m} p_{i_{j}}^{t_{j}}$, where at least one of the $i_{j}$ is in $\operatorname{Im} \psi$.

To demonstrate the positive application of Theorem 2.3, we present two nonintersecting classes of extensions satisfying (1.1) with countably many stable letters and solvable CP.

Theorem 2.7. If $G^{*}$ satisfies Assumptions 1.1 and $k_{i}=l_{i}, i \in \mathbb{N}$, then $G^{*}$ has solvable CP.

Proof. If $A^{*}=\left\{k_{i}, i \in \mathbb{N}\right\}$, then $A^{*}$ is recursive by assumption. Multiplication of idempotents in $S$ is given by $(a, a) \odot(b, b)=([a, b],[a, b])$, where $[a, b]$ is the least common multiple of $a$ and $b$. This proves that $(x, x) \in P\left(\mathbb{N}, \phi_{G^{*}}\right)$ if and only if, for some $n \geq 1, x$ is the l.c.m. of $n$ distinct elements of $A^{*}$. This can be decided by testing the divisors of $x$ for membership in $A^{*}$ and then testing the l.c.m. of each subset of the set of divisors in $A^{*}$ for equality with $x$. Hence, $P\left(\mathbb{N}, \phi_{G^{*}}\right)$ is recursive and the result follows from (2.3).

Theorem 2.8. If $G^{*}$ satisfies Assumptions 1.1 , the exponents are all distinct, and $k_{i} l_{i} \wedge k_{j} l_{j}=1$ whenever $i \neq j$, then $G^{*}$ has solvable $C P$. 
Proof (cf. [5, 3.6]). If $A=\left\{\left(k_{i}, l_{i}\right),\left(l_{i}, k_{i}\right), i \in \mathbb{N}\right\}$ and $\left(x_{1}, y_{1}\right),\left(x_{2}, y_{2}\right) \in$ $A$, with $\left(x_{2}, y_{2}\right) \neq\left(x_{1}, y_{1}\right)^{ \pm 1}$, then $\left(x_{1}, y_{1}\right) \odot\left(x_{2}, y_{2}\right)=\left(x_{2}, y_{2}\right) \odot\left(x_{1}, y_{1}\right)=$ $\left(x_{1} x_{2}, y_{1} y_{2}\right)$. Hence any product of elements in $A$ can be reordered so that for each index $i$ all elements with index $i$ occur together. Set $g_{i}=k_{i} \wedge l_{i}$, $K_{i}=k_{i} / g_{i}$, and $L_{i}=l_{i} / g_{i}$. Any product of powers of a fixed generator $\left(k_{i}, l_{i}\right)$, say, has a normal form $\left(g_{i} K_{i}^{r} K_{i}^{s} L_{i}^{t}, g_{i} K_{i}^{r} L_{i}^{s} L_{i}^{t}\right)^{ \pm 1}$, for some $r, s, t \geq$ 0 . Thus $(x, y) \in P\left(\mathbb{N}, \phi_{G^{*}}\right)$ if and only if, for some $n \geq 1,(x, y)=$ $\left(\prod_{i=1}^{n} g_{j_{i}} K_{j_{i}}^{r_{i}} P_{i}^{s_{i}} L_{j_{i}}^{t_{i}}, \prod_{i=1}^{n} g_{j_{i}} K_{j_{i}}^{r_{i}} Q_{i}^{s_{i}} L_{j_{i}}^{t_{i}}\right)$, where $r_{i}, s_{i}, t_{i} \geq 0$ and $P_{i}=K_{j_{i}}$ or $L_{j_{i}}$ when $Q_{i}=L_{j_{i}}$ or $K_{j_{i}}$, respectively, $1 \leq i \leq n$. Each divisor $d$ of $x$ can be tested for membership in the (recursive) exponent set, and if $d$ returns a positive answer, the unique $k_{i}$ (or $l_{i}$ ) it equals can be identified and the corresponding $l_{i}$ (or $k_{i}$ ) and $g_{i}$ found. Hence, it is possible to decide if $x$ is a product of powers of the required form. By relative primality, such a decomposition will be unique and can then be checked against $y$. Therefore, $P\left(\mathbb{N}, \phi_{G^{*}}\right)$ is recursive and the result follows from (2.3).

Relative primality conditions on the exponents have previously been used to obtain positive solutions of the CP for extensions with finitely many stable letters either directly $[1,5]$ or by a transformation of the $\mathrm{CP}$ to the word problem for a finitely generated commutative semigroup [2]. Lockhart's positive solution of the CP for extensions satisfying (1.1) with finitely many stable letters also uses a transformation of the $\mathrm{CP}$ to the word problem for a finitely generated commutative semigroup. For HNN extensions satisfying (1.1) with countably many stable letters, the $\mathrm{CP}$ transforms to the extended word problem for r.e. generated inverse subsemigroups of $S$. In the next section we identify the structure of $S$ and obtain a positive solution to the CP for a third class of extensions, in which all exponents are powers of a common base, in contrast to the class above.

\section{THE BICYCLIC SEMIGROUP}

We relate $S$ to the well-known bicyclic semigroup, $B$ (cf. [6, p. 144]). If we write $\mathbb{N}^{0}=\mathbb{N} \cup\{0\}$, then $B=\left(\mathbb{N}^{0} \times \mathbb{N}^{0}, \star\right)$, where the operation $\star$ is defined to be

$$
(a, b) \star(c, d)=(a+c-\min (b, c), b+d-\min (b, c)) .
$$

The complexity of the subsemigroup structure of $S$ becomes apparent when we prove it is isomorphic to $\bigoplus_{\omega} B$, the direct sum of countably many bicyclic semigroups. The ground set of $\bigoplus_{\omega} B$ is the set of infinite sequences of elements in $\mathbb{N}^{0} \times \mathbb{N}^{0}$ for which all but finitely many terms equal $(0,0)$, and its operation $\star$ is inherited positionwise from $B$.

Lemma 3.1. $S \cong \bigoplus_{\omega} B$.

Proof. For any $a \in \mathbb{N}$, let $e_{i}(a)$ be the exponent of the $i$ th prime $p_{i}$, in the representation of $a$ as a product of powers of distinct primes. For each $i \geq 1$, there is a semigroup homomorphism $\phi_{i}: S \rightarrow B$, given by $\phi_{i}(a, b)=$ $\left(e_{i}(a), e_{i}(b)\right)$. Define $\phi: S \rightarrow \bigoplus_{\omega} B$ by $\phi(a, b)=\left(\phi_{i}(a, b)\right)_{i=1}^{\infty}$. Then $\phi$ is a semigroup isomorphism.

The structure of the inverse subsemigroups of the bicyclic semigroup $B$ may be derived from the characterisation of Jones [7, 1.3]. In [4] we give a direct 
proof of this result and, consequently, a normal form for elements in such an edgepath semigroup.

Proposition 3.2 [7, 1.3; 4, 2.2 and 2.3]. Let $A$ be an inverse subsemigroup of $B$. Either $A$ is a set of idempotents or there exist integers $\delta \geq 1$ and $g_{1}<\cdots<$ $g_{n}<g_{1}+\delta \in \mathbb{N}^{0}$ and $a$ (possibly empty) set $\left\{a_{1}, \ldots, a_{m}\right\} \subseteq\left\{0, \ldots, g_{1}-1\right\}$, such that $\left\{\left(a_{j}, a_{j}\right), 1 \leq j \leq m ;\left(g_{i}, g_{i}+\delta\right), 1 \leq i \leq n\right\}$ generates $A$. In fact, in the latter case,

$$
A=\left\{\left(a_{j}, a_{j}\right), 1 \leq j \leq m\right\} \cup \bigcup_{i=1}^{n}\left\{\left(g_{i}+k \delta, g_{i}+l \delta\right), k, l \in \mathbb{N}^{0}\right\}
$$

That is, in an inverse subsemigroup $A$ of $B$ which is not an infinite set of idempotents, each element has a unique finite specification. Therefore, for every such $A$ there exists an algorithm which recognizes it, although there is no universal recursive way to construct such an algorithm from $A$.

Corollary 3.3. Unless it is a nonrecursive set of idempotents, an inverse subsemigroup of $B$ has solvable EWP.

To apply this corollary to the $\mathrm{CP}$, it remains only to link a class of HNN extensions to edgepath semigroups lying wholly in the bicyclic semigroup.

Lemma 3.4. Suppose $\phi_{\beta}: \mathbb{N} \rightarrow S$ has the form $\phi_{\beta}(j)=\left(\beta^{a_{j}}, \beta^{b_{j}}\right), a_{j}, b_{j} \in \mathbb{N}^{0}$, for a fixed integer $\beta \geq 2$. The mapping $\log _{\beta}: P\left(\mathbb{N}, \phi_{\beta}\right) \rightarrow B$ defined by

$$
\log _{\beta}\left(\left(\beta^{a}, \beta^{b}\right)\right)=(a, b)
$$

is a monomorphism of inverse semigroups.

Proof. $\log _{\beta}$ is easily checked to be a homomorphism of inverse semigroups, and the exponentiation map from $\mathbb{N}^{0}$ to $\mathbb{N}$ given by $n \mapsto \beta^{n}$ is one-to-one.

Since $\log _{\beta}$ is recursive when $\beta$ is known, the next result is an immediate consequence of the previous two.

Corollary 3.5. Unless $\operatorname{Im} \phi_{\beta}$ is a nonrecursive set of idempotents, $P\left(\mathbb{N}, \phi_{\beta}\right)$ is recursive. 2.3 .

Our final positive solution follows on combining this result with Theorem

Theorem 3.6. If $G^{*}$ satisfies Assumptions 1.1 and

$$
G^{*}=\left\langle G, t_{i} ; i \in I: x^{\beta^{a_{i}}}=t_{i}^{-1} x^{\beta^{b_{i}}} t_{i} ; a_{i}, b_{i} \in \mathbb{N}^{0}, i \in I\right\rangle
$$

then $G^{*}$ has solvable $C P$.

\section{REFERENCES}

1. M. Anshel, Conjugate powers in HNN groups, Proc. Amer. Math. Soc. 54 (1976), 19-23.

2. The conjugacy problem for HNN groups and the word problem for commutative semigroups, Proc. Amer. Math. Soc. 61 (1976), 223-224.

3. M. Anshel and K. McAloon, Reducibilities among decision problems for HNN groups, vector addition systems and subsystems of Peano arithmetic, Proc. Amer. Math. Soc. 89 (1983), 425-429. 
4. G. E. Farr and K. J. Horadam, Edgepath semigroups and the extended word problem (in preparation).

5. K. J. Horadam, The conjugacy problem for graph products with central cyclic edge groups, Proc. Amer. Math. Soc. 91 (1984), 345-350.

6. J. M. Howie, An introduction to semigroup theory, Academic Press, London, 1976.

7. P. R. Jones, The lattice of inverse subsemigroups of a reduced inverse semigroup, Glasgow Math. J. 17 (1976), 161-172.

8. J. M. Lockhart, An HNN-extension with cyclic associated subgroups and with unsolvable conjugacy problem, Trans. Amer. Math. Soc. 313 (1989), 331-345.

9. J. M. Lockhart, The conjugacy problem for graph products with infinite cyclic edge groups, Proc. Amer. Math. Soc. 117 (1993), 897-898.

10. H. Rogers, Jr., Theory of recursive functions and effective computability, McGraw-Hill, New York, 1968.

11. J.-P. Serre, Trees (translated by J. Stillwell), Springer-Verlag, Berlin, 1980.

Department of Mathematics, Royal Melbourne institute of Technology, G.P.O. Box 2476V, Melbourne, Victoria 3001, Australia

Cryptomathematics Research, Communications Division, Defense Science and TechNology Organisation, P. O. Box 1500, Salisbury, South Australia, 5108 Australia

Current address: Air Operations Division, Aeronautical Research Laboratory, 506 Lorimer St., Fishermens Bend, Victoria, 3207 Australia 